\title{
New osteoporosis guidelines for Canada
}

\author{
John A. Kanis MD \\ Previously published at www.cmaj.ca \\ $\infty \quad$ See related review article by Papaioannou and colleagues, page 1864
}

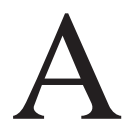

lthough osteoporosis has been documented for many years, the disease and the fractures that arise were commonly viewed as inevitable consequences of the aging process. The publication by the World Health Organization (WHO) of a report on the assessment of fracture risk and its application to screening for postmenopausal osteoporosis in 1994 provided diagnostic criteria for osteoporosis, based on the measurement of bone mineral density, and recognized osteoporosis as an established and well-defined disease affecting more than 75 million people in the United States, Europe and Japan.

Fractures due to osteoporosis commonly occur at the hip, spine, distal forearm and proximal humerus and are an important cause of mortality and morbidity in the Western world. In Sweden, the remaining lifetime risk of a fracture at the age of 50 years is $22 \%$ for men and $46 \%$ for women. This value for women is similar to that for coronary artery disease. ${ }^{2}$

Against this considerable burden, the good news is that osteoporosis can be treated, and a wide variety of agents is available for this purpose. Well-designed, prospective controlled studies have shown that many of these agents decrease the risk of vertebral fracture. Of these, some also reduce the risk of nonvertebral fractures, in some cases specifically at the hip. Unfortunately, a minority of patients in Canada (and elsewhere) are offered treatment, and of those for whom an intervention is prescribed, only a minority will adhere for any meaningful duration. ${ }^{3}$ The size of the problem, the availability of diagnostic tools and the advent of safe and effective treatments mean that the management of osteoporosis, with some exceptions, should devolve to primary care providers, if we are to manage the disease effectively. Hence, there is a need for practice guidelines that can be readily applied to primary care.

The 2010 clinical practice guidelines for the diagnosis and management of osteoporosis in Canada, appearing in this issue of the $C M A J,{ }^{4}$ provide a timely update of the evidence available on which to base guidelines that can be applied to the Canadian population. The review of the evidence is of high quality and is backed by much data derived from Canada. Levels of evidence and grades of recommendations from systematic reviews of all aspects of the management of osteoporosis are clearly presented. These new guidelines are timely because of important advances in the assessment of fracture risk that enable more effective targeting of treatments.

\section{Key points}

- Osteoporosis and fragility fractures are a major cause of morbidity and health care expenditure.

- Safe and effective treatments are available and the majority of cases can be managed in primary care.

- New guidelines provide updated evidence-based guidelines on diagnosis and management for Canada that can be applied to primary care.

The previous Canadian guidelines, ${ }^{5}$ published in 2002, directed treatments on the basis of thresholds in bone mineral density, in common with most guidance at the time. ${ }^{2}$ There are, however, limitations to using bone mineral density to determine thresholds for intervention. First, the T-score for bone mineral density decreases progressively with age. As such, among elderly patients, a T-score of -2.5 standard deviations (the threshold for osteoporosis) represents a higherthan-average bone mineral density and is associated with a correspondingly lower-than-average risk of fracture. Second, although bone mineral density has high specificity for predicting the risk of fracture, it has low sensitivity, so that the majority of osteoporotic fractures occur in individuals with bone mineral density values categorized as low risk. ${ }^{1}$

In the past 10 years, a great deal of research has identified factors other than bone mineral density that contribute to the risk of fracture. Examples include age, sex, prior fracture, family history of fracture and lifestyle risk factors such as physical inactivity and smoking. Some of these risk factors are partially or wholly independent of bone mineral density. Independent risk factors used in conjunction with bone mineral density enhance the information provided by bone mineral density alone. ${ }^{6}$ Conversely, some risk factors that are strongly dependent on bone mineral density alone can, in principle, be used to assess the risk of fracture in the absence of bone mineral density results. For this reason, the consideration of such risk factors improves fracture prognostication and the identification of individuals at high risk, who should have priority for treatment.

This shift in thinking resulted in the adoption, in 2005, of a system to assess absolute fracture risk, the Canadian Associa-

From the World Health Organization Collaborating Centre for Metabolic Bone Diseases, University of Sheffield, Sheffield, UK

CMAJ 2010. DOI:10.1503/cmaj.101438

All editorial matter in CMAJ represents the opinions of the authors and not necessarily those of the Canadian Medical Association. 
tion of Radiologists and Osteoporosis Canada tool (also known as CAROC). ${ }^{7}$ The guidelines include a description of the updated 2010 version of this system. The new guidelines take this concept a stage further in recommending use of FRAX, the fracture risk assessment tool of the WHO. This computer-based algorithm (available at www.shef.ac.uk/FRAX) provides country-specific models to assess the probability of fracture in men and women. ${ }^{2}$ Its extensive validation worldwide ${ }^{6}$ has included populations from Canada. ${ }^{8}$ The approach uses easily obtained clinical risk factors, either alone or with bone mineral density, to estimate the 10-year probability of fracture.

Application of this methodology to clinical practice, using either the Canadian version of FRAX or the 2010 version of the Canadian Association of Radiologists and Osteoporosis Canada tool, demands consideration of the fracture probability at which to intervene. There is no single formula for devising thresholds for intervention. The 10-year probability of fracture varies markedly in different countries, and thresholds also change with differences in costs, particularly the costs associated with fracture, which vary worldwide. There is also the issue of affordability or willingness to pay for a strategy. ${ }^{2}$ For all these reasons, it is important to define intervention and assessment thresholds on a country-bycountry basis, taking into account the setting for service provision and willingness to pay, as well as considerations of absolute costs.

The Canadian practice guidelines recommend that a 10-year probability of major osteoporotic fracture of greater than $20 \%$ be considered high risk, probability less than $10 \%$ be considered low risk and intermediate values be considered moderate risk. The high-risk threshold of $20 \%$ is consistent with the recommendation of the National Osteoporosis Foundation, which is based on cost-effectiveness analyses for the United States. ${ }^{9}$ It is expected that these intervention thresholds will target treatments to those with greatest need and, as importantly, avoid unnecessary treatment for the "worried well."

\section{This article was solicited and has not been peer reviewed.}

Competing interests: As director of the World Health Organization (WHO) Collaborating Centre for Metabolic Bone Diseases, Dr. Kanis led the team that developed the FRAX system for fracture risk assessment; he has no financial interest in FRAX. Dr. Kanis has served as a consultant to Abiogen, Italy; Amgen, USA, Switzerland and Belgium; Bayer, Germany; BesinsIscovesco, France; Biosintetica, Brazil; Boehringer Ingelheim, UK; Celtrix,
USA; D3A, France; the European Federation of Pharmaceutical Industries and Associations; Gador, Argentina; General Electric, USA; GSK, UK and USA; Hologic, Belgium and USA; Kissei, Japan; Leo Pharma, Denmark; Lilly, USA, Canada, Japan, Australia and UK; Merck Research Labs, USA; Merlin Ventures, UK; MRL, China; Novartis, Switzerland and USA; Novo Nordisk, Denmark; Nycomed, Norway; Ono, UK and Japan; Organon, Holland; Parke-Davis, USA; Pfizer, USA; Pharmexa, Denmark; Procter and Gamble, UK and USA; ProStrakan, UK; Roche, Germany, Australia, Switzerland and USA; Rotta Research, Italy; Sanofi-Aventis, USA; Schering, Germany and Finland. He has provided expert testimony for the High Court (UK). His institution has received grants from the Medical Research Council (UK), the Arthritis and Rheumatism Council (UK) and the European Federation of Pharmaceutical Industries and Associations. He has received speaker's fees from Amgen, Novartis and Servier. He has also worked with the European Society for Clinical and Economic Aspects of Osteoporosis and Osteoarthritis, the Government of Manitoba, the Group for the Respect of Ethics and Excellence in Science, INSERM (France), the Medical Research Council (UK), the Ministry of Public Health of China, the Ministry of Health of Australia, the National Institute for Health and Clinical Excellence (UK), the National Osteoporosis Guideline Group (UK), the National Osteoporosis Society (UK), the International Osteoporosis Foundation, the Japanese Osteoporosis Society, Osteoporosis 2000 (UK), Osteoporosis Australia, the Swiss Osteoporosis Society and the WHO.

\section{REFERENCES}

1. Assessment of fracture risk and its application to screening for postmenopausal osteoporosis. WHO Tech Rep Ser 843. Geneva (Switzerland): World Health Organization; 1994.

2. Kanis JA; World Health Organization Scientific Group. Assessment of osteoporosis at the primary health care level [technical report]. Sheffield (UK): University of Sheffield, WHO Collaborating Centre for Metabolic Bone Disease; 2007. Available: www.shef.ac.uk/FRAX/pdfs/WHO_Technical_Report.pdf (accessed 2010 Oct. 6).

3. Compston JE, Seeman E. Compliance with osteoporosis therapy is the weakest link. Lancet 2006;368:973-4.

4. Papaioannou A, Morin S, Cheung AM, et al.; Scientific Advisory Council of Osteoporosis Canada. 2010 clinical practice guidelines for the diagnosis and management of osteoporosis in Canada: summary. CMAJ 2010;182:1864-73.

5. Brown JP, Josse RG; Scientific Advisory Council of the Osteoporosis Society of Canada. 2002 clinical practice guidelines for the diagnosis and management of osteoporosis in Canada. CMAJ 2002;167(10 Suppl):S1-34.

6. Kanis JA, Oden A, Johnell O, et al. The use of clinical risk factors enhances the performance of BMD in the prediction of hip and osteoporotic fractures in men and women. Osteoporos Int 2007;18:1033-46.

7. Siminoski K, Leslie WD, Frame H, et al. Recommendations for bone mineral density reporting in Canada. Can Assoc Radiol J 2005;56:178-88.

8. Leslie WD, Lix LM, Johansson H, et al.; Manitoba Bone Density Pprogram. Independent clinical validation of a Canadian FRAX ${ }^{\circ}$ tool: fracture prediction and model calibration. J Bone Miner Res. 2010 Apr. 30 [Epub ahead of print].

9. Tosteson AN, Melton LJ 3rd, Dawson-Hughes B, et al. Cost-effective osteoporosis treatment thresholds: the United States perspective. Osteoporos Int 2008;19:437-47.

Correspondence to: Prof. John A. Kanis, WHO Collaborating Centre for Metabolic Bone Diseases, University of Sheffield Medical School, Beech Hill Road, Sheffield UK S10 2RX; w.j.Pontefract@shef.ac.uk 